Supporting Information

\title{
LiGaP $_{2} \mathrm{O}_{7}$ : A Potential UV Nonlinear Optical Crystal
}

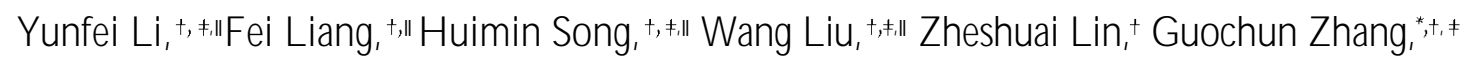
and Yicheng $\mathrm{Wu},{ }^{9}$

${ }^{+}$C enter for C rystal Research and D evelopment, Key Laboratory Functional C rystals and Laser T echnology of Chinese Academy of Sciences, Technical Institute of Physics and Chemistry, Chinese Academy of Sciences, Beijing 100190, P. R. C hina

${ }^{*}$ Center of M aterials Science and $O$ ptoelectronics Engineering, University of C hinese Academy of Sciences, Beijing 100049 , P. R. China

"U niversity of Chinese A cademy of Sciences, Beijing 100049, P. R. C hina

"Institute of Functional Crystal M aterials, T ianjin U niversity of T echnology, T ianjin 300384 P. R. China

Corresponding author: Guochun Zhang; gczhang@ mail.ipc.ac.cn.

Tel: 86-10-82543726; Fax: 86-10-82543726

\section{Experiment Content}

\section{Reagents}

$\mathrm{Li}_{2} \mathrm{CO}_{3}$ (Aladdin, 99\%), $\mathrm{Ga}_{2} \mathrm{O}_{3}$ (Aladdin, 99.99\%), and $\mathrm{NH}_{4} \mathrm{H}_{2} \mathrm{PO}_{4}$ (Sinopharm, 99.5\%) were used as received.

\section{Solid- states synthesis and single crystal growth}

Polycrystalline samples of $\mathrm{LiGaP}_{2} \mathrm{O}_{7}$ were synthesized by the traditional solid-state reaction techniques by mixing $\mathrm{Li}_{2} \mathrm{CO}_{3}, \mathrm{Ga}_{2} \mathrm{O}_{3}$, and $\mathrm{NH}_{4} \mathrm{H}_{2} \mathrm{PO}_{4}$ with the molar ratio of $0.8: 1: 2$. The mixtures were ground thoroughly, heated to $300{ }^{\circ} \mathrm{C}$ at a rate of $10{ }^{\circ} \mathrm{C} / \mathrm{h}$ to decompose the $\mathrm{CO}_{2}$ and $\mathrm{NH}_{3}$ completely, and then sintered at this temperature for $24 \mathrm{~h}$. Subsequently, the products were cooled to room temperature, ground thoroughly. Finally, the products were sintered at $850{ }^{\circ} \mathrm{C}$ for $120 \mathrm{~h}$. With this procedure, pure polycrystalline of $\mathrm{LiGaP}_{2} \mathrm{O}_{7}$ were successfully obtained. The phase purity was confirmed by powder X-ray diffraction (XRD) analysis, which was carried out at room temperature on a Bruker D8 Focus X-ray diffractometer equipped with a diffracted monochromatic set for $\mathrm{Cu} \mathrm{K} \alpha$ $\left(\lambda=1.5418 \AA\right.$ ) radiation. The scanning step width of $0.02^{\circ}$ and scanning rate of $0.05^{\circ-1}$ were applied to record the patterns in the $2 \theta$ range of $10-70^{\circ}$. The measured powder XRD pattern was found to be in good agreement with the calculated ones based on the single crystal crystallographic data. (Figure 1 in manuscript).

Single crystals of $\mathrm{LiGaP}_{2} \mathrm{O}_{7}$ were grown with $\mathrm{Li}_{2} \mathrm{O}_{-} \mathrm{P}_{2} \mathrm{O}_{5}$ flux system by a spontaneous nucleation approach. A mixture of $\mathrm{Li}_{2} \mathrm{CO}_{3}, \mathrm{Ga}_{2} \mathrm{O}_{3}$, and $\mathrm{NH}_{4} \mathrm{H}_{2} \mathrm{PO}_{4}$ according to a molar ratio of 2:1:5 was thoroughly ground and placed into a platinum crucible. Similarly, the crucible was heated to $300{ }^{\circ} \mathrm{C}$ at a rate of $10^{\circ} \mathrm{C} / \mathrm{h}$ to decompose the $\mathrm{CO}_{2}$ and $\mathrm{NH}_{3}$ completely. Then the crucible was rapidly heated to $950{ }^{\circ} \mathrm{C}$ in a vertical, programmable temperature controlled growth furnace. It was hold at this temperature for $10 \mathrm{~h}$ to ensure that the mixture melt thoroughly. Then the melt was slowly cooled down to $850^{\circ} \mathrm{C}$ at a rate of $2{ }^{\circ} \mathrm{C} / \mathrm{h}$. The yield of $\mathrm{LiGaP}_{2} \mathrm{O}_{7}$ is above $70 \%$ under such optimized raw material 
ratio. Several colorless block crystals were separated mechanically from the crucible for the further characterization by single-crystal XRD measurements.

\section{Structure Determination}

A colorless $\mathrm{LiGaP}_{2} \mathrm{O}_{7}$ crystal of $0.1 \times 0.08 \times 0.05 \mathrm{~mm} 3$ was selected for single-crystal XRD analysis. Samples were mounted on a glass fiber for data collection on a Rigaku AFC10 diffractometer equipped with a graphite-monochromated $\mathrm{K} \alpha(\lambda=0.71073 \AA)$ radiation at $153.15 \mathrm{~K}$ and a Saturn CCD detector. CrystalClear program was used to record the intensity data and to conduct cell refinement and data reduction. The structure was solved by the direct method with program SHELXS and refined with the least-squares program SHELXL. The final refinement includes anisotropic displacement parameter. The solved structures were checked by the program PLATON and no higher symmetric were found.

\section{Thermal Analysis}

Thermal gravimetric (TG) analysis and differential scanning calorimetric (DSC) of the sample $\mathrm{LiGaP}_{2} \mathrm{O}_{7}$ were investigated on a simultaneous thermal analyzer instrument. The experiment was carried out with the synthesized $\mathrm{LiGaP}_{2} \mathrm{O}_{7}$ powder. The measurement was carried out in an atmosphere of flowing $\mathrm{N}_{2}$. About $11 \mathrm{mg}$ of $\mathrm{LiGaP}_{2} \mathrm{O}_{7}$ was placed in a Pt crucible, and heated at a rate of $10^{\circ} \mathrm{C} / \mathrm{min}$ from room temperature to $1000{ }^{\circ} \mathrm{C}$, and then cooled to room temperature at the same rate.

\section{Infrared Spectrum}

The infrared spectrum was recorded on a FTIR Biorad spectrometer FTS-40A with dynamic alignment. The samples were mixed with $\mathrm{KBr}$, and then examined in a range of $400-4000 \mathrm{~cm}^{-1}$ with a resolution of $2 \mathrm{~cm}^{-1}$.

\section{UV-Vis-NIR Diffuse Reflectance Spectrum}

The UV-visible near IR (UV-vis-NIR) diffuse reflection data were recorded at room temperature using a SolidSpec-3700 DUV spectrophotometer in the wavelength range from 200 to $1600 \mathrm{~nm}$.

\section{Powder Second Harmonic Generation Measurements}

The powder SHG measurements for targeted I and II were carried out by the Kurtz-Perry method. The measurement were performed with a Q-switched Nd:YAG laser at 1064nm. Fine single crystals of $\mathrm{LiGaP}_{2} \mathrm{O}_{7}$ were ground and sieved into the following particle size ranges: 20-40, 41-75, 76-100, 101-150, and 151-200 $\mu \mathrm{m}$. Single crystals with different particle sizes were pressed between glass microscope cover slides and secured in $1 \mathrm{~mm}$ thick aluminum holders containing an 8-mm diameter hole. These holders were placed in a light-tight box and irradiated with the laser. The intensities of the frequency-doubled output emitted from the samples were collected by a photomultiplier tube. The commercial KDP crystals were also ground and sieved into the same particle size range for reference.

\section{Calculation Methods}

The electronic structure calculations for $\mathrm{LiGaP}_{2} \mathrm{O}_{7}$ were performed using CASTEP package ${ }^{1}$ based on density functional theory (DFT). ${ }^{2}$ The generalized gradient-corrected exchange-correlation function (GGA) developed by Perdew, Burke, and Ernzerhof (PBE) ${ }^{3}$ were adopted. The interactions between the ionic cores and electrons were described by norm-conserving pseudopotentials. ${ }^{4}$ The 
following orbital electrons were treated as valence electrons: $\mathrm{Li} 2 s^{1}$, Ga $3 d^{10} 4 s^{2} 4 p^{1}, \mathrm{P} 3 s^{2} 3 p^{3}$, and $\mathrm{O}$ $2 s^{2} 2 p^{4}$. The number of plane waves included in the basis was determined by a cutoff energy of $900 \mathrm{eV}$, and the numerical integration of the Brillouin zone was performed using a $6 \times 6 \times 4$ Monkhorst-Pack scheme $k$-point grids. The second-order SHG coefficients were calculated by the formula developed by Lin et. $a l,{ }^{5}$ which has been applied in many NLO oxides successfully. ${ }^{6-7}$

Figure S1 XRD patterns after melting of $\mathrm{LiGaP}_{2} \mathrm{O}_{7}$ powder samples

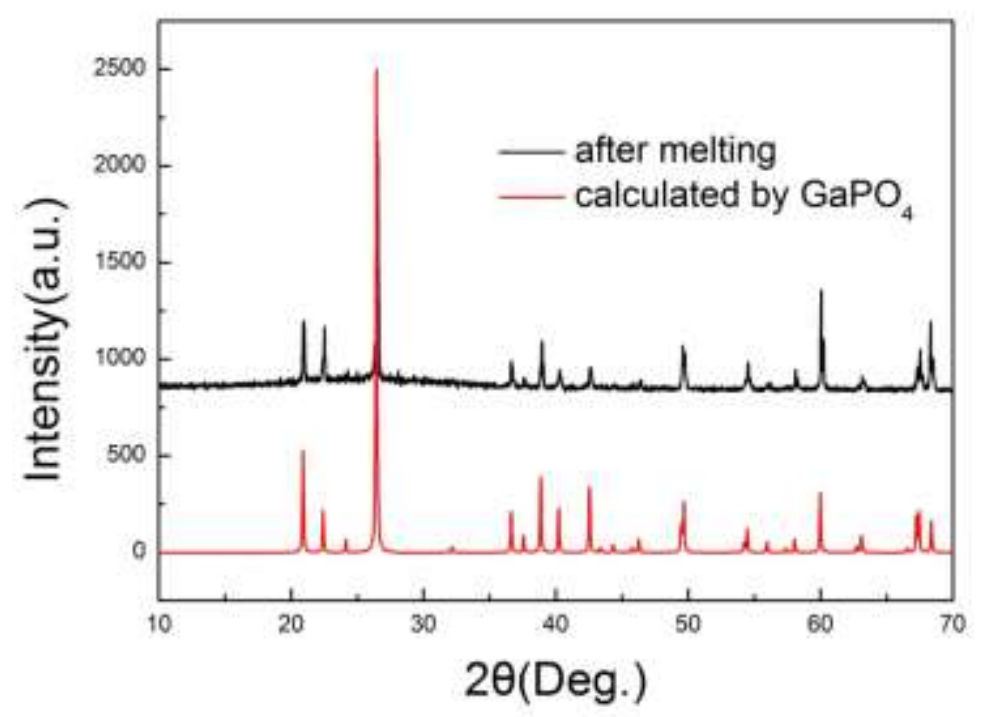

Figure S2 $\left[\mathrm{P}_{2} \mathrm{O}_{7}\right]$ dimer in $\mathrm{LiGaP}_{2} \mathrm{O}_{7}$

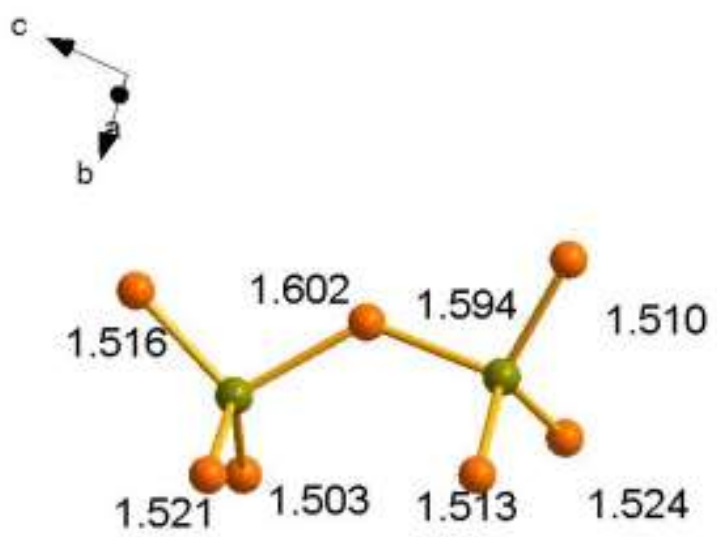


Figure S3 Coordination environment of Li atom

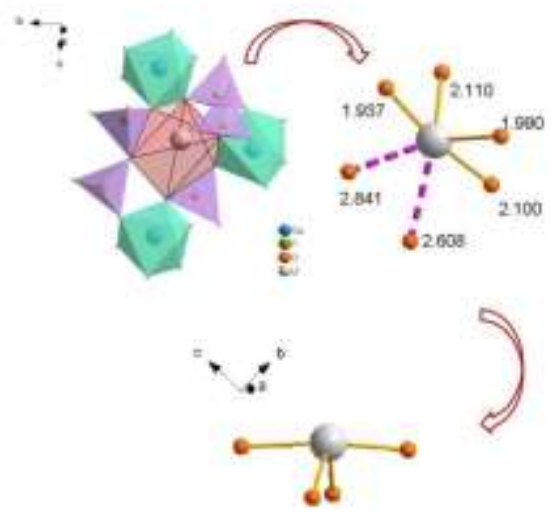

Figure S4 Comparison of band structures for $\mathrm{LiGaP}_{2} \mathrm{O}_{7}, \mathrm{LiFeP}_{2} \mathrm{O}_{7}$, and $\mathrm{LiCrP}_{2} \mathrm{O}_{7}$
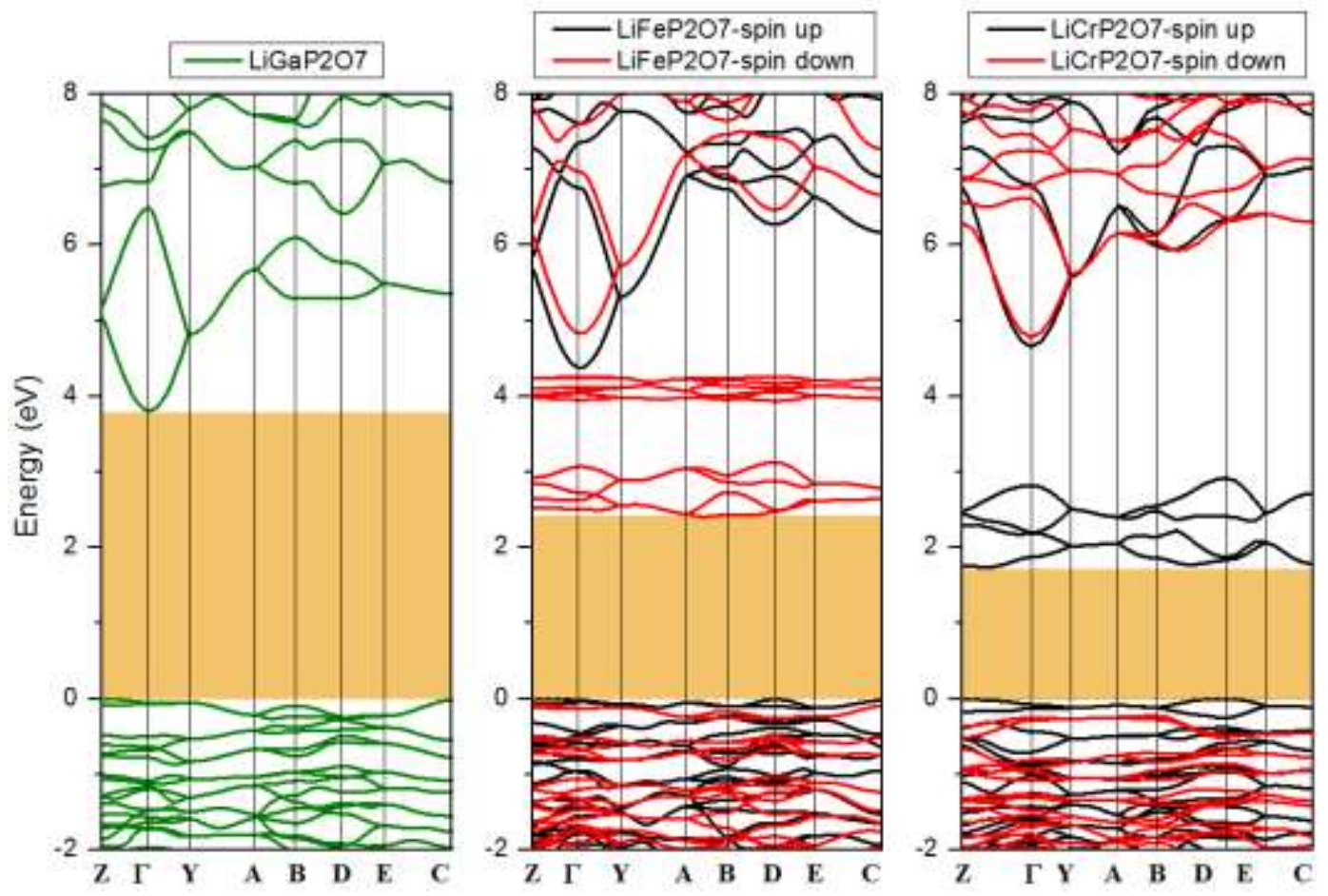
Table S1 Crystal data and structure refinements for $\mathrm{LiGaP}_{2} \mathrm{O}_{7}$.

\begin{tabular}{|c|c|}
\hline Empirical formula & $\mathrm{LiGaP}_{2} \mathrm{O}_{7}$ \\
\hline Formula weight & 250.60 \\
\hline Temperature/K & 153.1500 \\
\hline Crystal system & monoclinic \\
\hline Space group & $P 2_{1}$ \\
\hline $\mathrm{a} / \AA$ & $4.7593(10)$ \\
\hline $\mathrm{b} / \AA$ & $7.9586(16)$ \\
\hline $\mathrm{c} / \AA$ & $6.8940(14)$ \\
\hline$\beta /{ }^{\circ}$ & $109.06(3)$ \\
\hline Volume $/ \AA^{3}$ & $246.81(10)$ \\
\hline $\mathrm{Z}$ & 2 \\
\hline$\rho_{\text {calc }} \mathrm{g} / \mathrm{cm}^{3}$ & 3.372 \\
\hline$\mu / \mathrm{mm}^{-1}$ & 6.199 \\
\hline $\mathrm{F}(000)$ & 240.0 \\
\hline Crystal size $/ \mathrm{mm}^{3}$ & $0.1 \times 0.08 \times 0.05$ \\
\hline Radiation & Molybdenum $(\lambda=0.710747)$ \\
\hline $2 \Theta$ range for data collection $/{ }^{\circ}$ & 8.084 to 54.936 \\
\hline Index ranges & $\begin{array}{c}-6 \leq \mathrm{h} \leq 6,-10 \leq \mathrm{k} \leq 9 \\
-8 \leq 1 \leq 8\end{array}$ \\
\hline Reflections collected & 2032 \\
\hline Independent reflections & $1092\left[\mathrm{R}_{\text {int }}=0.0532, \mathrm{R}_{\text {sigma }}=0.0832\right]$ \\
\hline Data/restraints/parameters & $1092 / 1 / 45$ \\
\hline Goodness-of-fit on $\mathrm{F}^{2}$ & 1.128 \\
\hline Final $R$ indexes $[\mathrm{I}>=2 \sigma(\mathrm{I})]$ & $\mathrm{R}_{1}=0.0552, \mathrm{wR}_{2}=0.0977$ \\
\hline Final R indexes [all data] & $\mathrm{R}_{1}=0.0625, \mathrm{wR}_{2}=0.1019$ \\
\hline Flack parameter & $0.05(3)$ \\
\hline
\end{tabular}

Table S2 Fractional atomic coordinates $\left(\times 10^{4}\right)$ and equivalent isotropic displacement parameters $\left(\AA^{2} \times 10^{3}\right)$ for $\mathrm{LiGaP}_{2} \mathrm{O}_{7}$. $\mathrm{U}_{\text {eq }}$ is defined as $1 / 3$ of the trace of the orthogonallized $\mathrm{U}_{\mathrm{ij}}$ tensor

\begin{tabular}{cccccc}
\hline Atom & $x$ & $y$ & $z$ & $\mathrm{U}(\mathrm{eq})$ & BVS \\
\hline $\mathrm{Ga}$ & $-2209(3)$ & $7318(2)$ & $2659.4(19)$ & $4.9(3)$ & 3.195 \\
$\mathrm{P} 1$ & $2064(8)$ & $5137(4)$ & $862(5)$ & $4.1(7)$ & 5.023 \\
P2 & $6018(7)$ & $4142(4)$ & $4841(5)$ & $5.6(7)$ & 5.021 \\
O6 & $3988(19)$ & $4146(12)$ & $6154(13)$ & $5.9(19)$ & 2.030 \\
O7 & $8202(19)$ & $2703(11)$ & $5261(13)$ & $7(2)$ & 2.097 \\
O3 & $1310(20)$ & $4212(11)$ & $-1167(13)$ & $3.0(18)$ & 2.047 \\
O5 & $7490(20)$ & $5837(11)$ & $4923(13)$ & $5.1(18)$ & 1.987 \\
O2 & $3930(20)$ & $6679(11)$ & $818(14)$ & $6.6(19)$ & 1.873 \\
O1 & $-650(20)$ & $5540(12)$ & $1433(14)$ & $8(2)$ & 1.967 \\
O4 & $4030(20)$ & $3826(12)$ & $2514(14)$ & $7.1(19)$ & 2.117 \\
Li & $1920(50)$ & $6100(30)$ & $6880(30)$ & $6(5)$ & 0.879 \\
\hline
\end{tabular}


Table S3 Selected bond lengths for $\mathrm{LiGaP}_{2} \mathrm{O}_{7}$.

\begin{tabular}{|c|c|c|c|c|c|}
\hline Atom & Atom & Length/Å & Atom & Atom & Length/Å \\
\hline $\mathrm{Ga}$ & $\mathrm{O}^{1}$ & $1.988(9)$ & P2 & O6 & $1.524(9)$ \\
\hline $\mathrm{Ga}$ & $\mathrm{O} 7^{2}$ & $2.000(9)$ & P2 & O7 & $1.510(9)$ \\
\hline $\mathrm{Ga}$ & $\mathrm{O} 3^{3}$ & $1.950(9)$ & $\mathrm{P} 2$ & O5 & $1.512(9)$ \\
\hline $\mathrm{Ga}$ & $\mathrm{O}^{4}$ & $1.998(9)$ & $\mathrm{P} 2$ & $\mathrm{O} 4$ & $1.594(9)$ \\
\hline $\mathrm{Ga}$ & $\mathrm{O} 2^{4}$ & $1.930(9)$ & O7 & $\mathrm{Li}^{6}$ & $1.94(2)$ \\
\hline $\mathrm{Ga}$ & $\mathrm{O} 1$ & $1.918(10)$ & $\mathrm{O} 3$ & $\mathrm{Li}^{5}$ & $2.10(2)$ \\
\hline $\mathrm{P} 1$ & $\mathrm{O} 3$ & $1.516(9)$ & O5 & $\mathrm{Li}^{7}$ & $2.11(2)$ \\
\hline $\mathrm{P} 1$ & $\mathrm{O} 2$ & $1.520(10)$ & $\mathrm{O} 2$ & $\mathrm{Li}^{5}$ & 2.61(2) \\
\hline $\mathrm{P} 1$ & $\mathrm{O} 1$ & $1.502(10)$ & & & \\
\hline P1 & $\mathrm{O} 4$ & $1.602(10)$ & & & \\
\hline
\end{tabular}

${ }^{1}-\mathrm{X}, 1 / 2+\mathrm{Y}, 1-\mathrm{Z} ;{ }^{2} 1-\mathrm{X}, 1 / 2+\mathrm{Y}, 1-\mathrm{Z} ;{ }^{3}-\mathrm{X}, 1 / 2+\mathrm{Y},-\mathrm{Z} ;{ }^{4}-1+\mathrm{X},+\mathrm{Y},+\mathrm{Z} ;{ }^{5}+\mathrm{X},+\mathrm{Y},-1+\mathrm{Z} ;{ }^{6} 1-\mathrm{X},-1 / 2+\mathrm{Y}, 1-\mathrm{Z} ;{ }^{7} 1+\mathrm{X},+\mathrm{Y},+\mathrm{Z}$

Table S4 Selected bond angles for $\mathrm{LiGaP}_{2} \mathrm{O}_{7}$.

\begin{tabular}{|c|c|c|c|c|c|c|c|}
\hline Atom1 & Atom2 & Atom3 & Angle ${ }^{\circ}$ & Atom1 & Atom2 & Atom3 & Angle ${ }^{\circ}$ \\
\hline $\mathrm{O}^{1}$ & $\mathrm{Ga}$ & $\mathrm{O}^{2}$ & $91.6(4)$ & $\mathrm{O} 3$ & $\mathrm{P} 1$ & $\mathrm{O} 2$ & $109.8(5)$ \\
\hline $\mathrm{O} 6^{1}$ & $\mathrm{Ga}$ & $O 5^{3}$ & $87.8(4)$ & $\mathrm{O} 3$ & $\mathrm{P} 1$ & $\mathrm{O} 4$ & $104.3(5)$ \\
\hline $\mathrm{O}^{4}$ & $\mathrm{Ga}$ & $\mathrm{O} 7^{2}$ & $86.4(4)$ & $\mathrm{O} 2$ & $\mathrm{P} 1$ & $\mathrm{O} 4$ & $109.3(5)$ \\
\hline $\mathrm{O}^{4}$ & $\mathrm{Ga}$ & $\mathrm{O} 5^{3}$ & $162.3(4)$ & O1 & $\mathrm{P} 1$ & $\mathrm{O} 3$ & $112.3(5)$ \\
\hline $\mathrm{O} 5^{3}$ & $\mathrm{Ga}$ & $\mathrm{O} 7^{2}$ & $80.0(4)$ & O1 & $\mathrm{P} 1$ & $\mathrm{O} 2$ & $112.9(6)$ \\
\hline $\mathrm{O} 2^{3}$ & $\mathrm{Ga}$ & $\mathrm{O} 6^{1}$ & $91.2(4)$ & O1 & $\mathrm{P} 1$ & $\mathrm{O} 4$ & $107.8(5)$ \\
\hline $\mathrm{O} 2^{3}$ & $\mathrm{Ga}$ & $\mathrm{O} 7^{2}$ & $172.8(4)$ & O1 & $\mathrm{P} 1$ & $\mathrm{O} 2$ & $112.9(6)$ \\
\hline $\mathrm{O} 2^{3}$ & $\mathrm{Ga}$ & $\mathrm{O} 3^{4}$ & $100.6(4)$ & O6 & $\mathrm{P} 2$ & $\mathrm{O} 4$ & $108.4(5)$ \\
\hline $\mathrm{O} 2^{3}$ & $\mathrm{Ga}$ & $\mathrm{O} 5^{3}$ & $93.5(4)$ & $\mathrm{O} 7$ & $\mathrm{P} 2$ & O6 & $114.7(5)$ \\
\hline $\mathrm{O} 1$ & $\mathrm{Ga}$ & $\mathrm{O} 6^{1}$ & $177.7(4)$ & O7 & $\mathrm{P} 2$ & O5 & $112.9(5)$ \\
\hline O1 & $\mathrm{Ga}$ & $\mathrm{O}^{2}$ & $90.7(4)$ & $\mathrm{O} 7$ & $\mathrm{P} 2$ & $\mathrm{O} 4$ & $102.6(5)$ \\
\hline O1 & $\mathrm{Ga}$ & $\mathrm{O} 3^{4}$ & $98.9(3)$ & O5 & $\mathrm{P} 2$ & O6 & $110.5(5)$ \\
\hline O1 & $\mathrm{Ga}$ & $05^{3}$ & $92.7(4)$ & O5 & $\mathrm{P} 2$ & $\mathrm{O} 4$ & $107.0(5)$ \\
\hline O1 & $\mathrm{Ga}$ & $\mathrm{O} 2^{3}$ & $86.5(4)$ & & & & \\
\hline $\mathrm{O} 7^{2}$ & $\mathrm{Li}$ & $\mathrm{O} 5^{3}$ & $78.6(9)$ & O6 & $\mathrm{Li}$ & $\mathrm{O} 3^{10}$ & 77.7(9) \\
\hline $\mathrm{O} 7^{2}$ & $\mathrm{Li}$ & $\mathrm{O} 2^{10}$ & $125.9(11)$ & O6 & $\mathrm{Li}$ & $\mathrm{O} 5^{3}$ & $103.6(11)$ \\
\hline $\mathrm{O} 3^{10}$ & $\mathrm{Li}$ & $\mathrm{O} 5^{3}$ & 91.1(9) & O6 & $\mathrm{Li}$ & $\mathrm{O} 2^{10}$ & $111.0(10)$ \\
\hline $\mathrm{O} 3^{10}$ & $\mathrm{Li}$ & $\mathrm{O} 2^{10}$ & $62.6(7)$ & $\mathrm{O} 7^{2}$ & $\mathrm{Li}$ & O6 & $102.6(10)$ \\
\hline $\mathrm{O} 5^{3}$ & $\mathrm{Li}$ & $\mathrm{O} 2^{10}$ & $129.2(10)$ & $\mathrm{O} 7^{2}$ & $\mathrm{Li}$ & $\mathrm{O} 3^{10}$ & $169.5(14)$ \\
\hline
\end{tabular}

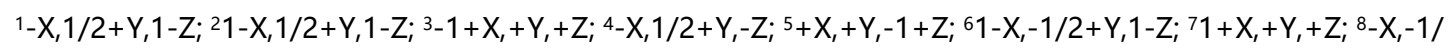
$2+Y, 1-Z ;{ }^{9}-X,-1 / 2+Y,-Z ;{ }^{10}+X,+Y, 1+$ 


\section{REFERENCE}

1. Clark, S. J.; Segall, M. D.; Pickard, C. J.; Hasnip, P. J.; Probert, M. J.; Refson, K.; Payne, M. C., First principles methods using CASTEP. Z. Kristallogr. 2005, 220 (5-6), 567-570.

2. Kohn, W., Nobel Lecture: Electronic structure of matter-wave functions and density functionals. Rev. Mod. Phys. 1999, 71 (5), 1253-1266.

3. Perdew, J. P.; Burke, K.; Ernzerhof, M., Generalized gradient approximation made simple. Phys. Rev. Lett. 1996, 77 (18), 3865-3868.

4. Vanderbilt, D., Soft Self-consistent Pseudopotentials in a Generalized Eigenvalue Formalism. Phys. Rev. B 1990, 41 (11), 7892-7895.

5. Lin, J.; Lee, M. H.; Liu, Z. P.; Chen, C. T.; Pickard, C. J., Mechanism for linear and nonlinear optical effects in beta-BaB ${ }_{2} \mathrm{O}_{4}$ crystals. Phys. Rev. B 1999, 60 (19), 13380-13389.

6. Liang, F.; Kang, L.; Zhang, X.; Lee, M.; Lin, Z.; Wu, Y., Molecular Construction Using $\left(\mathrm{C}_{3} \mathrm{~N}_{3} \mathrm{O}_{3}\right)^{3-}$ Anions: Analysis and Prospect for Inorganic Metal Cyanurates Nonlinear Optical Materials. Cryst. Growth \& Des. 2017, 17 (7), 4015-4020.

7. Luo, M.; Liang, F.; Song, Y.; Zhao, D.; Xu, F.; Ye, N.; Lin, Z., $\mathrm{M}_{2} \mathrm{~B}_{10} \mathrm{O}_{14} \mathrm{~F}_{6}(\mathrm{M}=\mathrm{Ca}$, Sr): Two Noncentrosymmetric Alkaline Earth Fluorooxoborates as Promising Next-Generation Deep-Ultraviolet Nonlinear Optical Materials. J. Am. Chem. Soc. 2018, 140 (11), 3884-3887. 